\title{
Endoscopic Ultrasonography-guided Fine-needle Aspiration Revealed Metastasis-induced Acute Pancreatitis in a Patient with Adrenocortical Carcinoma
}

\author{
Toshitaka Mori ${ }^{1}$, Hiromu Kondo ${ }^{1}$, Itaru Naitoh ${ }^{2}$, Tetsuo Koyama ${ }^{1}$, Yuya Takenaka ${ }^{1}$, \\ Hirohiko Komai ${ }^{1}$, Sachiko Araki ${ }^{1}$, Mika Kitagawa ${ }^{2}$, Nobuhiro Nishigaki ${ }^{1}$, Yoshito Tanaka ${ }^{1}$, \\ Keisuke Itoh ${ }^{1}$, Chihiro Hasegawa ${ }^{1}$, Takashi Kawai ${ }^{1}$ and Kazuki Hayashi ${ }^{2}$
}

\begin{abstract}
:
A 26-year-old woman complained of upper abdominal pain. Computed tomography (CT) showed acute pancreatitis, a left adrenal tumor and solitary right pulmonary metastasis. She underwent left adrenalectomy; the adrenal tumor was diagnosed as adrenocortical carcinoma (ACC). When preparing to resect the pulmonary metastasis, she suffered a second acute pancreatic attack. Magnetic resonance cholangiopancreatography (MRCP) showed that the proximal main pancreatic duct (MPD) was dilated, and the distal MPD was diminished; however, no pancreatic tumor was observed on CT or MRCP. Endoscopic ultrasonography revealed a solitary pancreatic mass, which was diagnosed as pancreatic metastasis from ACC by endoscopic ultrasonography-guided fine-needle aspiration.
\end{abstract}

Key words: metastasis-induced acute pancreatitis, adrenocortical carcinoma, endoscopic ultrasonographyguided fine-needle aspiration

(Intern Med 58: 2645-2649, 2019)

(DOI: 10.2169/internalmedicine.2450-18)

\section{Introduction}

Adrenocortical carcinoma (ACC) is a rare and highly aggressive malignancy with an annual incidence of 0.7-2.0 cases per million individuals. The initial manifestations of ACC are Cushing's syndrome and/or virilization presenting as an abdominal mass or the feeling of abdominal fullness. Acute pancreatitis has never been reported as an initial manifestation of ACC.

There are various causes of acute pancreatitis, including alcohol, choledocholithiasis, hypertriglyceridemia, pancreatic divisum, sphincter of Oddi dysfunction, and pancreatic tumors. Metastasis-induced acute pancreatitis (MIAP) is very rare and initially manifests as cancer. In cases of MIAP, the original cancer is usually in the lung, especially small cell lung cancer (SCLC), although renal cell carcinoma has also been reported.

We herein report the first case of a patient with ACC presenting as an initial manifestation of MIAP and reveal that endoscopic ultrasonography-guided fine-needle aspiration (EUS-FNA) can be used to diagnose metastasis from ACC.

\section{Case Report}

A 26-year-old woman visited our hospital with upper abdominal pain. Her medical history and family history were not remarkable. She worked as a bar hostess and so consumed alcohol habitually (>100 g ethanol per day) and smoked.

Computed tomography (CT) showed a left adrenal tumor (110×77 mm in diameter), and imaging revealed acute pancreatitis (enlargement of the pancreatic parenchyma and stranding of the surrounding fat). Her serum amylase (1,084

${ }^{1}$ Department of Gastroenterology, Nagoya City East Medical Center, Japan and ${ }^{2}$ Department of Gastroenterology and Metabolism, Nagoya City University Graduate School of Medical Sciences, Japan

Received: November 29, 2018; Accepted: March 19, 2019; Advance Publication by J-STAGE: June 7, 2019

Correspondence to Dr. Hiromu Kondo, hkondo@higashi-hosp.jp 

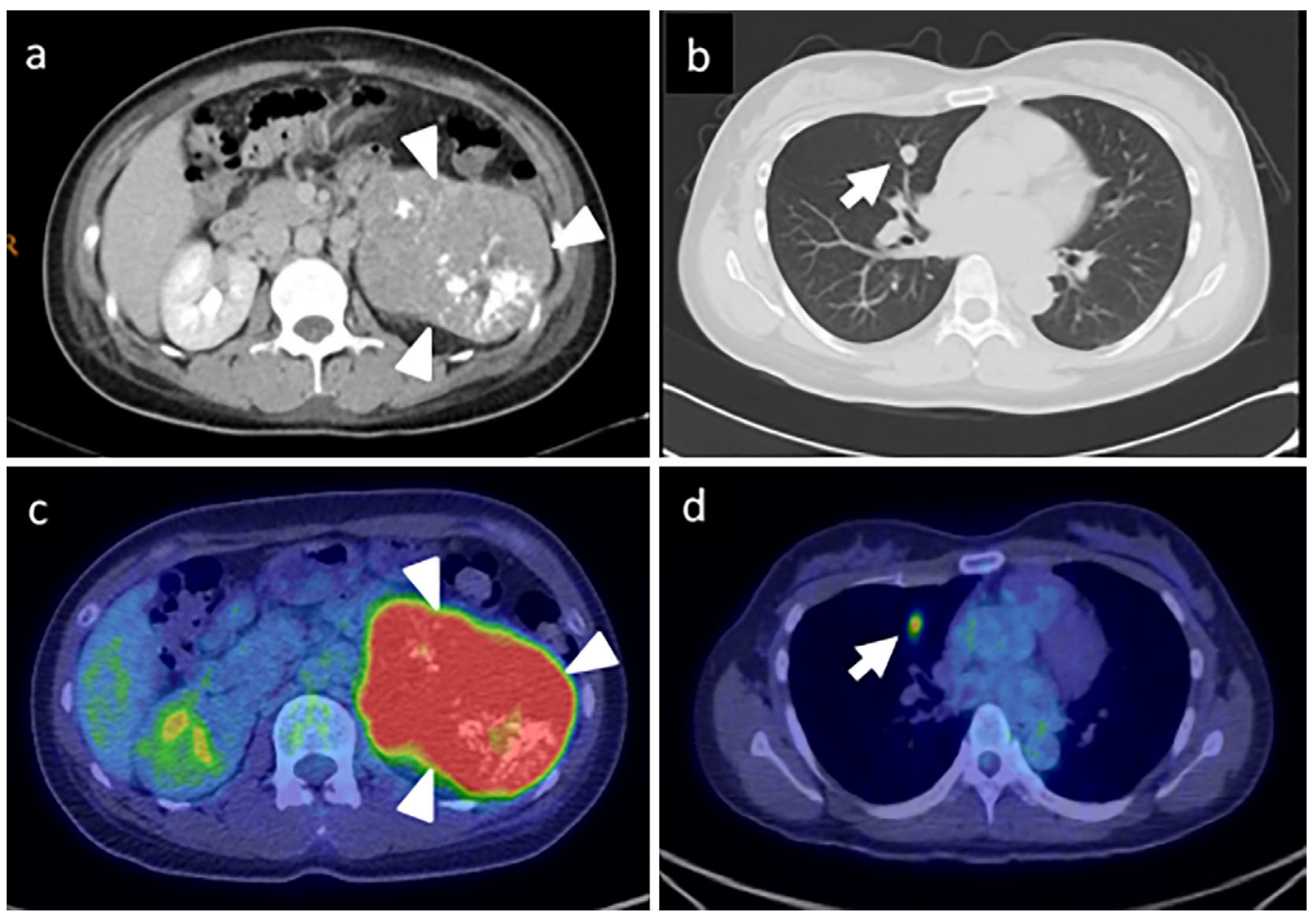

Figure 1. a, b) Contrast-enhanced computed tomography (CT) showing a large left adrenal tumor with heterogeneous enhancement (arrowhead) and a right pulmonary tumor (arrow). c, d) Positron 18F-fluorodeoxyglucose positron emission tomography with CT showing both tumors, with maximum standardized uptake values of 9.5 and 3.9.

$\mathrm{U} / \mathrm{L}$; normal range: 37.0-125 U/L), white blood cell count $\left(9,940 / \mathrm{mm}^{3}\right.$; normal range: $\left.3,500-8,000 / \mathrm{mm}^{3}\right)$, and Creactive protein $(18.0 \mathrm{mg} / \mathrm{dL}$; normal range: $0.0-0.3 \mathrm{mg} / \mathrm{dL})$ values were elevated. We diagnosed her with acute pancreatitis caused by alcohol and an incidental adrenal tumor. To diagnose the adrenal tumor, we conducted an endocrinologic examination. She had no typical symptoms of Cushing's syndrome, such as moon face or central obesity, pheochromocytoma, or virilization. There was no ${ }^{123}$ I metaiodobenzylguanidine uptake in the left adrenal tumor, and urine metanephrine and normetanephrine levels were within the normal range. Serum adrenocorticotropic hormone (ACTH) levels were low $(<1.0 \mathrm{pg} / \mathrm{mL}$; normal range: $7.2-63.3 \mathrm{pg} /$ $\mathrm{mL}$ ), and dehydroepiandrosterone sulfate levels were high $(2,493 \mu \mathrm{g} / \mathrm{dL}$; normal range: 92-399 $\mu \mathrm{g} / \mathrm{mL})$. There was a lack of circadian rhythm of cortisol, and overnight low-dose dexamethasone $(1 \mathrm{mg})$ suppression tests revealed a lack of cortisol suppression (before, $18.4 \mu \mathrm{g} / \mathrm{dL}$; after 19.4, $\mu \mathrm{g} / \mathrm{dL}$ ).

Examinations revealed that she had non-ACTH-dependent Cushing's syndrome, not pheochromocytoma. Thereafter, we performed contrast-enhanced CT and positron emission tomography (PET) studies, which revealed a left adrenal tumor $[93 \times 78 \times 78.5 \mathrm{~mm}$; maximum standardized uptake value $\left.\left(\mathrm{SUV}_{\max }\right), 9.5\right]$ with a solitary right lung metastasis $(10 \times 9.8$ $\mathrm{mm} ; \mathrm{SUV}_{\max }$, 3.9) (Fig. 1).

After her condition recovered with intravenous infusion, she underwent adrenalectomy with open surgery on hospital day 8. A pathologic examination revealed that the adrenal tumor was ACC according to the Weiss system. The tumor met 5 of the 9 criteria for ACC [mitotic rate $>5 / 50$ highpower fields, $<25 \%$ clear cells, diffuse architecture $(>1 / 3$ of the tumor), necrosis, and sinusoidal invasion], and the Ki67/ MIB1 labeling index (Ki67/MIB1LI) of the tumor was $56 \%$ (Fig. 2).

While preparing for pulmonary resection, she suffered a second upper abdominal attack on hospital day 22. Laboratory data showed that her serum amylase level and white blood cell count had increased $\left(1,629 \mathrm{U} / \mathrm{L}\right.$ and $11,260 / \mathrm{mm}^{3}$, respectively). CT showed acute pancreatitis and proximal main pancreatic duct (MPD) dilatation. Magnetic resonance cholangiopancreatography (MRCP) also revealed proximal MPD dilatation and diminishment of the distal MPD; however, neither imaging modality revealed a distal pancreatic mass (Fig. 3). Therefore, we performed EUS to examine the pancreas on hospital day 27 , which revealed a solitary pancreatic mass ( $8 \mathrm{~mm}$ in diameter) that obstructed the proximal pancreatic duct. Subsequently, we performed EUS-FNA with a 22-G needle (EZ shot 3 plus; Olympus, Tokyo, Japan) (Fig. 4). Pathologic findings revealed a pancreatic mass that was a solitary pancreatic metastasis from ACC (Fig. 5). We diagnosed the patient with acute pancreatitis attacks manifesting as MIAP. To prevent recurrent acute pancreatitis caused by pancreatic metastasis, we performed endoscopic pancreatic stenting (EPS) via the minor papilla through the MPD on hospital day 35. Thereafter, she did not experience acute pancreatitis, but the pancreatic metastasis increased in 

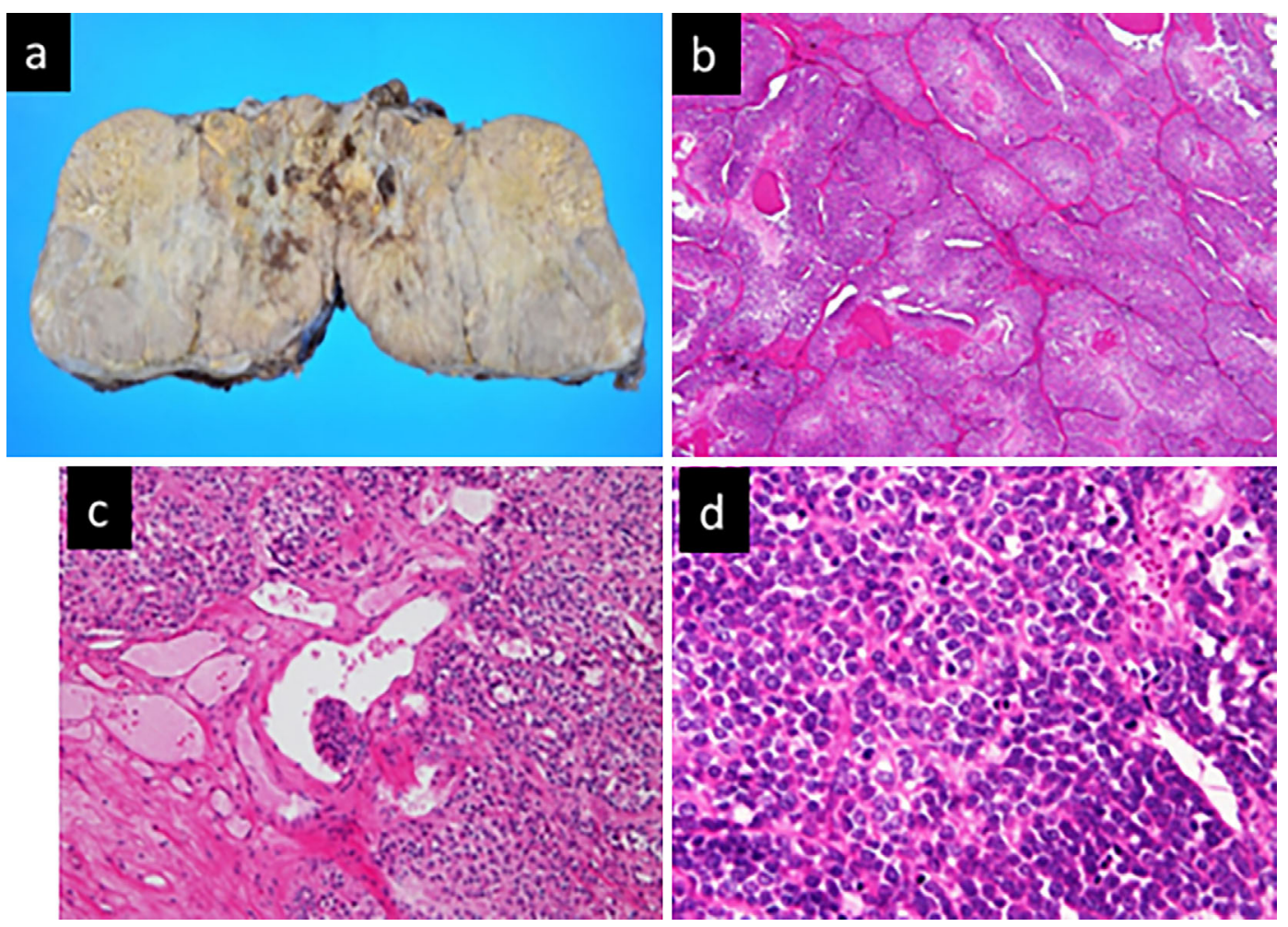

Figure 2. Adrenocortical carcinoma of the left adrenal gland. a: Resected specimen. b: Clusters of tumor cells with eosinophilic cytoplasm and $<25 \%$ clear cells [Hematoxylin and Eosin (H\&E) staining, $\times 40]$. c: Sinusoidal invasion $(H \& E$ staining, $\times 200)$. d: Mitotic rate $>5 / 50$ high-power fields $(H \& E$ staining, $\times 400)$.
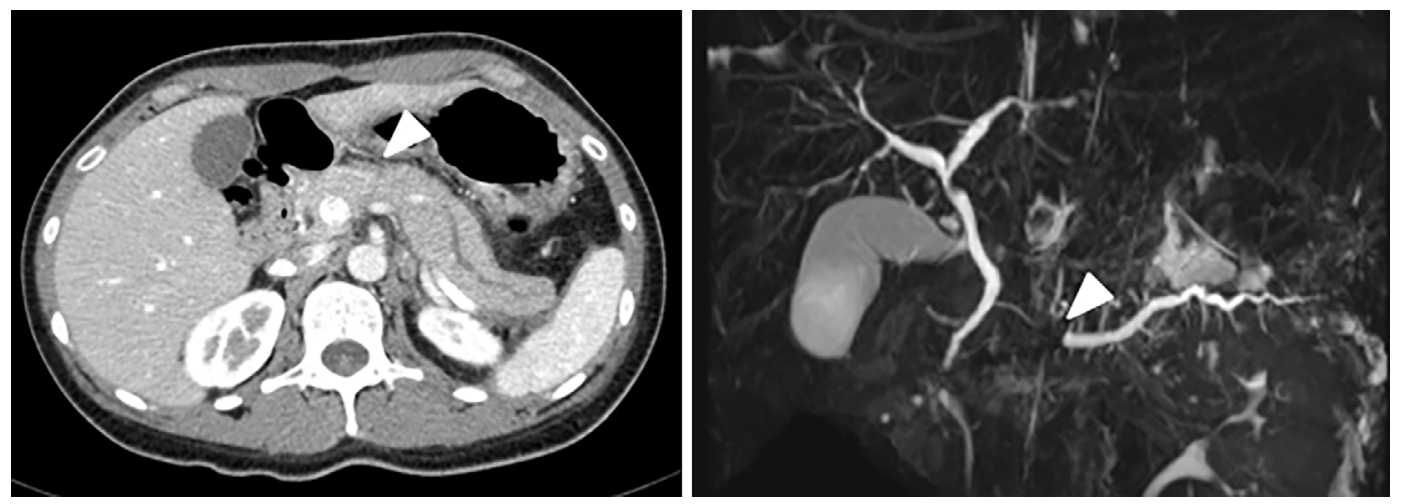

Figure 3. CT and magnetic resonance imaging showing pancreatic dilatation in the pancreatic body and tail, which was not seen at admission. However, there was no obvious tumor in the pancreatic parenchyma (arrowhead).

size. Synchronous multiple metastases from ACC required the initiation of mitotane-based chemotherapy (etoposide, doxorubicin, cisplatin, and mitotane) without resection of other organs. She underwent 12 cycles of mitotane-based chemotherapy, however, she ultimately died of the progression of ACC at 26.5 months after the first admission.

\section{Discussion}

This case is the first report of MIAP from ACC that was diagnosed by EUS-FNA according to a search of the PubMed database using the key words "metastasis-induced acute pancreatitis", "adrenocortical carcinoma" and "endoscopic ultrasonography-guided fine-needle aspiration". ACC is a rare and highly aggressive malignancy, with an annual incidence of 0.7-2.0 cases per million individuals. ACC can occur at any age but has a peak incidence at 40-50 years, and women are more often affected than men, accounting for $55-60 \%$ of all cases (1).

Complete surgical resection is the only potentially curative treatment for ACC (2). However, metastasis is common in patients with ACC, with approximately one-third of patients having synchronous metastasis at the diagnosis (3). The most common metastatic sites are lung, liver, and bone. 

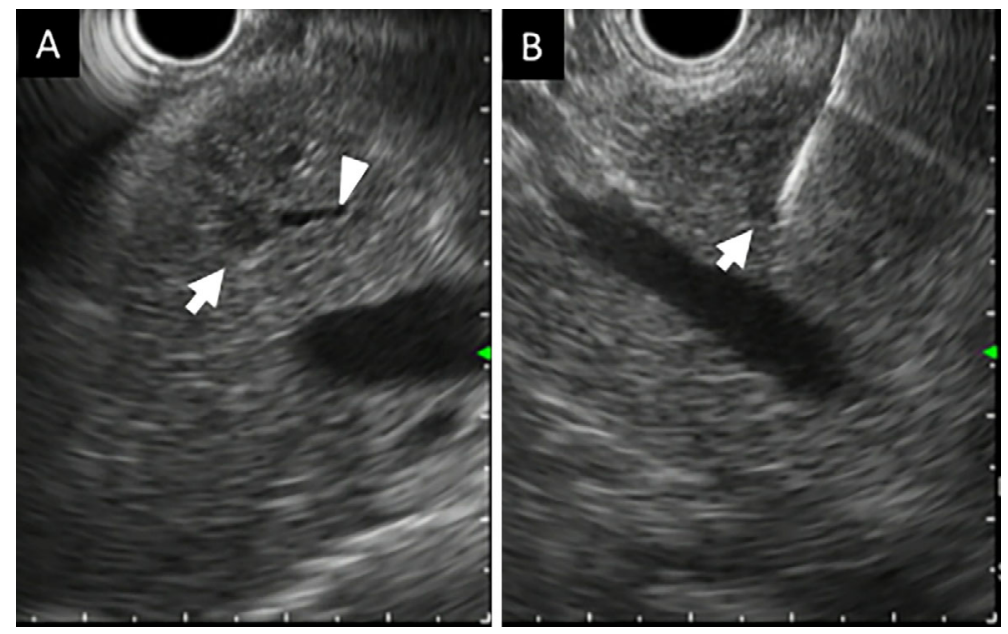

Figure 4. A: Endoscopic ultrasonography revealing a pancreatic tumor ( $8 \mathrm{~mm}$ in diameter) in the pancreatic body (arrow). Arrowhead shows the pancreatic main duct. B: Needle penetrating the tumor.
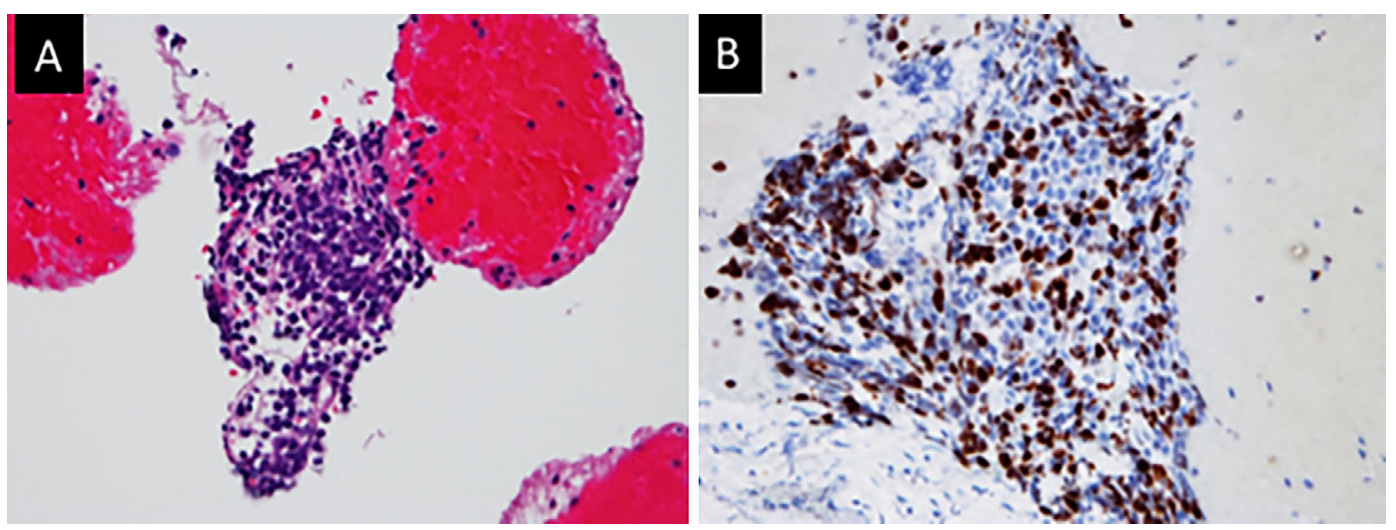

Figure 5. A: Microscopically, the tumor cells had eosinophilic cytoplasm and mimicked the adrenocortical carcinoma. B: The Ki67/MIB1 labeling index of the specimen was $55 \%$.

After initial resection, locoregional recurrence becomes a challenge with pelvic, peritoneal, or retroperitoneal metastases (4). In this case, liver metastasis was found three months after adrenalectomy, and she ultimately died of multiorgan disfunction from liver and lung metastases and peritoneal dissemination.

Pancreatic metastasis is rare compared with liver and lung metastasis. Adsay et al. reported secondary tumors of the pancreas in $81(1.6 \%)$ of 4,955 adult autopsy cases and 38 (3.9\%) of 973 surgical cases (5). The lung (34 cases), gastrointestinal tract [stomach (10), colon (5), esophagus (4), rectum (1)], kidney (4), breast (3), and liver (2) were the most frequent sites of primary in the autopsy series, and most were non-Hodgkin's lymphomas (11) in the surgical series (5).

According to a large single-center experience of diagnosing pancreatic metastases with EUS-FNA, the primary tumor sites were the kidney (21 cases), lung (8), skin (6), colon (4), breast (3), small bowel (2), stomach (2), liver (1), ovary (1), and bladder (1) in 49 patients. In addition, EUS-FNA confirmed pancreatic metastases in $47 / 49$ patients $(95.9 \%)$ without complications (6). The adrenal gland was not mentioned as a secondary tumor of the pancreas.

Baur et al. reported a case of pancreatic metastasis from ACC. Their case was one of recurrent ACC with a solitary pancreatic metastasis that occurred six years after resection of the primary tumor (7).

The current case was MIAP. Two weeks before the second attack of acute pancreatitis, PET-CT showed no proximal pancreatic duct dilatation or pancreatic mass. We did not perform MRCP at the first attack of acute pancreatitis because we considered the etiology to have been alcohol, given the patient's history of alcohol intake. We might have been able to detect narrowing of the MPD or slight upstream dilatation had we performed MRCP after the first attack. At the second attack, CT and MRCP revealed proximal pancreatic duct dilatation but no solitary pancreatic mass. In contrast, EUS showed an obvious solitary pancreatic mass and EUS-FNA revealed pancreatic metastasis from ACC on pathological findings.

EUS is now regarded as the most sensitive imaging modality for the detection of pancreatic lesions. According to a 
systematic review, the overall sensitivities of EUS, CT, US, and MRI for the detection of pancreatic tumors were $94 \%$, $74 \%, 67 \%$, and $79 \%$, respectively (8). For the detection of pancreatic tumor $\leq 20 \mathrm{~mm}$, EUS had a higher sensitivity than contrast-enhanced CT $(94.4 \%$ vs. $50.0 \%, n=36)(9)$. Furthermore, EUS-FNA is the best modality for obtaining a tissue diagnosis; it has a sensitivity of $85-86.8 \%$ and specificity of $95.8-98 \%$ for determining the etiology of a solid pancreatic mass $(10,11)$.

There are no standard treatment approaches for MIAP due to the rarity of this disease and its poor prognosis. However, several authors reported that MIAP in patients with SCLC was treated successfully with chemotherapy to achieve pancreatic metastasis shrinkage and loss without recurrent acute pancreatitis $(12,13)$. In the current case, ACC showed poor responsiveness to chemotherapy and radiotherapy; therefore, we inserted a pancreatic stent through the pancreatic metastasis from the minor papilla and decompressed the proximal pancreatic duct, similar to the EPS performed for MPD strictures in patients with chronic pancreatitis (14). On reviewing the literature, Oku et al. reported that EPS was previously performed for MIAP to prevent recurrent acute pancreatitis (15).

In the current case, we were unable to determine whether alcohol or pancreatic metastasis caused the first attack of pancreatitis. However, her proximal MPD was dilated slightly on CT performed after the first attack, and had had no episodes of acute pancreatitis despite being a heavy drinker. Therefore, we suspect that pancreatic metastasis caused the acute pancreatitis.

In conclusion, we should consider the possibility of pancreatic tumor when we encounter cases of acute pancreatitis with uncertain causes. EUS is a useful modality for detecting small pancreatic masses, and we emphasize the efficacy of EUS-FNA for the diagnosis of pancreatic metastasis.

The authors state that they have no Conflict of Interest (COI).

\section{References}

1. Fassnacht M, Kroiss M, Allolio B. Update in adrenocortical carcinoma. J Clin Endocrinol Metab 98: 4551-4564, 2013.

2. Allolio B, Hahner S, Weismann D, Fassnacht M. Management of adrenocortical carcinoma. Clin Endocrinol (Oxf) 60: 273-287,
2004.

3. Fassnacht M, Johanssen S, Quinkler M, et al. Limited prognostic value of the 2004 International Union Against Cancer staging classification for adrenocortical carcinoma: proposal for a Revised TNM Classification. Cancer 115: 243-250, 2009.

4. Else T, Kim AC, Sabolch A, et al. Adrenocortical carcinoma. Endocr Rev 35: 282-326, 2014.

5. Adsay NV, Andea A, Basturk O, Kilinc N, Nassar H, Cheng JD. Secondary tumors of the pancreas: an analysis of a surgical and autopsy database and review of the literature. Virchows Archi 444: 527-535, 2004

6. El H II, LeBlanc JK, Sherman S, et al. Endoscopic ultrasoundguided biopsy of pancreatic metastases: a large single-center experience. Pancreas 42: 524-530, 2013.

7. Baur J, Schedelbeck U, Pulzer A, et al. A case report of a solitary pancreatic metastasis of an adrenocortical carcinoma. BMC Surg 15: 93, 2015

8. Kitano $\mathrm{M}$, Yoshida $\mathrm{T}$, Itonaga $\mathrm{M}$, Tamura $\mathrm{T}$, Hatamaru $\mathrm{K}$, Yamashita Y. Impact of endoscopic ultrasonography on diagnosis of pancreatic cancer. J Gastroenterol 54: 19-32, 2019.

9. Sakamoto H, Kitano M, Suetomi Y, Maekawa K, Takeyama Y, Kudo M. Utility of contrast-enhanced endoscopic ultrasonography for diagnosis of small pancreatic carcinomas. Ultrasound Med Biol 34: 525-532, 2008.

10. Hewitt MJ, McPhail MJ, Possamai L, Dhar A, Vlavianos P, Monahan KJ. EUS-guided FNA for diagnosis of solid pancreatic neoplasms: a meta-analysis. Gastrointest Endosc 75: 319-331, 2012.

11. Puli SR, Bechtold ML, Buxbaum JL, Eloubeidi MA. How good is endoscopic ultrasound-guided fine-needle aspiration in diagnosing the correct etiology for a solid pancreatic mass?: a meta-analysis and systematic review. Pancreas 42: 20-26, 2013.

12. Tanaka H, Nakazawa $T$, Yoshida $M$, et al. Metastasis-induced acute pancreatitis in a patient with small cell carcinoma of the lungs. JOP 10: 557-561, 2009.

13. Okutur K, Bozkurt M, Korkmaz T, et al. Metastasis-induced acute pancreatitis successfully treated with chemotherapy and radiotherapy in a patient with small cell lung cancer. Case Rep Oncol Med 2015: 304279, 2015.

14. Dumonceau JM, Delhaye M, Tringali A, et al. Endoscopic treatment of chronic pancreatitis: European Society of Gastrointestinal Endoscopy (ESGE) Clinical Guideline. Endoscopy 44: 784-800, 2012.

15. Oku T, Maeda M, Wada $Y$, et al. Successful management of metastasis-induced pancreatitis by endoscopic pancreatic duct stenting in a case of large cell lung cancer. Dig Endosc 19: 134$138,2007$.

The Internal Medicine is an Open Access journal distributed under the Creative Commons Attribution-NonCommercial-NoDerivatives 4.0 International License. To view the details of this license, please visit (https://creativecommons.org/licenses/ by-nc-nd/4.0/).

\footnotetext{
(C) 2019 The Japanese Society of Internal Medicine Intern Med 58: 2645-2649, 2019
} 